\title{
The latent structure and reliability of the emotional trait section of the Affective and Emotional Composite Temperament Scale (AFECTS)
}

\author{
Hudson W. de Carvalho ${ }^{1}$ \\ https://orcid.org/0000-0001-8360-5053 \\ Hugo Cogo-Moreira ${ }^{2}$ \\ https://orcid.org/0000-0001-9411-9237 \\ KAREN JANSEN ${ }^{3}$ \\ https://orcid.org/0000-0003-3494-8070 \\ LUCIANO SOUZA 3 \\ https://orcid.org/0000-0001-9965-4837 \\ JERÔNIMO BRANCO 3 \\ https://orcid.org/0000-0001-6750-2740 \\ RICARDO SILVA 3 \\ https://orcid.org/0000-0002-9114-7037 \\ Diogo R. LARA ${ }^{4}$ \\ https://orcid.org/0000-0002-6690-9490 \\ Received: 02/23/2019 - Accepted: 01/14/2020 \\ DOl: 10.1590/0101-60830000000225 \\ 'Department of Psychology, Federal University of Pelotas, Pelotas, RS, Brazil. \\ 2 Graduate Program in Psychiatry and Medical Psychology, Federal University of São Paulo, São Paulo, SP, Brazil. \\ ${ }_{3}^{3}$ Postgraduate Program in Health and Behavior, Catholic University of Pelotas, Pelotas, RS, Brazil. \\ ${ }_{4}$ Faculty of Biosciences, Pontifical Catholic University of Rio Grande do Sul, Porto Alegre, RS, Brazil.
}

\begin{abstract}
Background: The Emotional and Affective Composite Temperament (AFECT) model describes originally six traits of volition, anger, inhibition (fear and caution subordinate factors), control, sensitivity, and coping. However, fear and caution have shown opposite relatioships with criteria-variables, indicating factor independence. Objective: The current investigation aimed to advance in the evaluation of the psychometric properties of the emotional trait section of the Emotional and Affective Composite Temperament Scale (AFECTS) by examining the suitability of a 7-factor structure and the reliability of each scale using data from a population-based sample. Methods: AFECTS was administered via face-to-face assessments in a single-session, population-based cross-sectional survey. Samples was composed of teenagers and adults (14 to 35 years). The latent structure and reliability were analyzed via structural equation modeling: confirmatory factor analysis was used to test the a priori correlated 7-factor model (with fear and caution designed as single-factors) and trait-scores reliability was assessed by the estimation of information curves. Results: Findings attested the suitability of the 7-factor model presumed to underline the item set of the traits section of AFECTS and information curve interpretation showed adequate levels of reliability for all trait-scores. Discussion: The 7-factor model showed robust indicators of construct validity for the AFECTS.
\end{abstract}

Carvalho HW et al. / Arch Clin Psychiatry. 2020;47(1):25-9

Keywords: Temperament, personality, psychological traits, structuctural equation modeling, psychometrics.

\section{Introduction}

The Affective and Emotional Composite Temperament model ${ }^{1}$ (AFECT) is a revised and expanded version of the Fear and Anger model 2,3 . Originally, the Fear and Anger model conceived temperament with two independent traits of emotional activation (drive and anger) and inhibition (fear and caution) ${ }^{2,3}$. This bifactor model had many implications for the understanding of psychopathology: it, in fact, anticipated a basic framework of predisposition to most mental disorders, which included a conceptual map to understand comorbidity patterns ${ }^{2,3}$. The vectors of activation and inhibition were also designed to tap specific neuroanatomical, neurochemical, and genetic undepinings of behavior and to inform psychopharmacological treatments ${ }^{2}$. Explicitly, the aim of the Fear and Anger model was to offer a conceptual framework that could inform clinical assessment and therapeutics to mental heath professionals.

Nevertheless, this bidimensional model was unable to account for neuropsychological domains keen for the understanding of emotion regulation, including the functions accountable for the modulation of activating and inhibiting behavior. The Fear and Anger model was too parcimounious to offer a comprehensive understanding of psychological adjustment trajectories in non-clinical contexts. Thus, the AFECT model ${ }^{1}$ was developed to engender a general theory of behavior that could comprise basic motivation features (activation and inhibition) with psychological functions related to self-regulation (control), vulnerability (emotional sensitivity), and resilience (coping).

In the AFECT model, ${ }^{1}$ activation is described by two relatively independent factors of volition and anger: The first is related to positive emotionality and positive engagement, while the second is linked to intense emotion manifestations and aggressiveness ${ }^{1}$.

Inhibition ${ }^{1}$ was designed as a second-order factor that accounted for the correlations between fear an caution first-order factors. Nevertheless, accumulating evidence have shown that fear and caution display opposite association with external outcome criteria: while fear is investilly associated with psychosocial adjustment patterns, caution seem to predict positive adjustment ${ }^{4-7}$. Thus, in this investigation, we addressed Inhibition vector as comprised by two single-factors of fear and caution: fear is thought to arises from "here and now" threaten situations and is related to freezing and flight reactions. Caution inhibits behavior by increasing attention bias to potential environment harms $\mathbf{s}^{1,2}$. 
Control is conceived as a single emotional trait responsible for promoting the adaptation of one's behavior to the environment and to one's cognitive goals by modulating the levels of activation (volition and anger) and inhibition (caution and fear). Thus, it is a self- and context-monitoring dimension related to executive/frontal circuitry ${ }^{1}$.

Sensitivity is a single trait that describes the extent to which someone is vulnerable to interpersonal and environmental stress and harm. On the other hand, coping is a single trait that aims to predict one's ability to deal positively with harmful experiences ${ }^{1}$.

The traits of the AFECT model can be assessed using the emotional section of the Affectivite and Emotional Composite Temperament Scale (AFECTS). This section is composed of 48 selfreport items that are assessed via a 7-point bipolar in Likert scale. Its validation study ${ }^{1}$ corroborated the 6 -factor model (inhibion was designed as a second-order factor with two subfactors of fear and caution) believed to underline AFECTS item set. Each factor displayed excellent level of internal consistency reliability ${ }^{1}$. Psychometric findings were considered to be robust once goodness of fit indexes were satisfactory and the data from a large and heterogeneous community sample was available ${ }^{1}$.

The AFECTS underwent a process of cultural adaptation and validation to Mexico ${ }^{8}$ using a sample of 350 participants from the general population and of 91 stable outpatients with various psychiatric diagnoses. Factor structure replicated the a priori sixfactor structure with excellent levels of internal consistency reliability. Traits scores also discriminated the general population sample from the clinical one 8 .

Other studies have shown that AFECTS trait scores differentiated individuals in regard to dissimilar traumatic courses ${ }^{4}$, sexual orientation identities ${ }^{5}$, substance use and misuse patterns ${ }^{7,9}$, personality disorders ${ }^{10}$, and daily energy patterns and cronotypes ${ }^{11}$. These findings showed that higher scores on volition, caution, control and coping were associated with more adaptative outcomes and social privilege, while higher scores on the traits anger, fear, and sensitivity were correlated with maladaptative outcomes and social vulnerability. Taken together, these findings attest positively the construct validity status of the AFECTS emotional section and indicate that fear and caution may be better understood as sigle-factors each.

Most studies using the AFECTS rely on Internet based data collection. Internet mediated studies have many advantages, such as the possibility to gather large samples at low cost or to increase data reliability when assessing sensitive issues, such as substance use or sexual behavior ${ }^{12,13}$. Nonetheless, some limitations are also present: samples tend to be biased to higher socioeconomic status, women, and highly motivated participants ${ }^{12}$. Thus, the current investigation aims to advance in the psychometric evaluation of the emotional section of the AFECTS using a representative and probabilistic samples of adolescents and adults that responded to the AFECTS via a traditional data collection methodology (face-to-face interview). As aforementioned, because fear and caution have shown opposite empirical relationships with external criteria, we tested the validity of a structural model based on 7 latent factors that allegedly underlines AFECTS item intercorrelations.

\section{Methods}

\section{Ethics}

The ethics committee of the Catholic University of Pelotas approved the protocol (ETHICS PROTOCOL: 15/2010) of the current study. Repondents agreed to participate and signed the free and informed consent form. This form was shaped to achieve the requirements of the National Health Council of Brazil (Resolution 196/1996) and the Code of Ethics of the World Medical Association (Declaration of Helsinki). Participants who were identified to have any mental disorder were assigned to a psychological and psychiatric assistance in a mental health ambulatory of the Catholic University of Pelotas with no cost.

\section{Participants and procedures}

The data set of the current study was produced by a single-session, population-based cross-sectional survey carried out in the urban area of Pelotas - a city located in the extreme south of Brazil. The target population was composed of individuals from both sexes with age ranging from 14 to 35 years.

Cluster sampling was achieved following the demographic data provided by the Brazilian Institute of Geography and Statistics (IBGE, 2008). This census divided the urban zone of the city of Pelotas into 448 sections with a target population of about 97,000 individuals aged 14-35. Out of these, 89 census sections were randomly selected and, subsequently, 2,756 residents were randomly identified. Participants were first contacted by telephone to explain the research goals, motivate participation, and schedule a data collection session. In total, 143 out of the 2,756 residents refused to take part in the study and other 265 were not found.

The resulting sample included 2,344 participants: 1,273 women (54.3\% women) and $1,071(45.7 \%)$ men. Mean age was of 24.1 (SD $=6.1$ ) years, most participants declared to be Caucasian $(75.3 \%)$, single $(66.7 \%)$, employed $(51.9 \%)$, and to have $11.3(\mathrm{SD}=3.3)$ years of formal education. The demographic profile of the sample is detailed in Table 1.

Table 1. Sociodemographic characteristics of the sample

\begin{tabular}{|l|c|c|c|c|}
\hline \multicolumn{2}{|c|}{} & Women & Men & Total \\
\hline Age & Mean (SD) & $24.37(5.99)$ & $23.86(6.05)$ & $24.14(6.02)$ \\
\hline Ethnicity & Caucasian & $976(76.7 \%)$ & $79.7(74.4 \%)$ & $1,773(75.3 \%)$ \\
& Afrodescendent & $169(13.3 \%)$ & $133(12.4 \%)$ & $302(12.9 \%)$ \\
& Asian & $06(0.5 \%)$ & $09(0.8 \%)$ & $15(0.6 \%)$ \\
& Amerindian & $05(0.4 \%)$ & $13(1.2 \%)$ & $18(0.8 \%)$ \\
& Other & $117(9.2 \%)$ & $119(11.1 \%)$ & $236(10.1 \%)$ \\
\hline Education & Basic & $737(57.9 \%)$ & $589(55.8 \%)$ & $1,335(57 \%)$ \\
& High-School & $370(29.1 \%)$ & $319(29.8 \%)$ & $689(86.3 \%)$ \\
& University & $166(13 \%)$ & $154(14.8 \%)$ & $320(13.7 \%)$ \\
\hline Marital & Single & $812(63.8 \%)$ & $749(69.9 \%)$ & $1,561(66.7 \%)$ \\
status & Married & $427(33.5 \%)$ & $306(28.6 \%)$ & $733(31.3 \%)$ \\
& Widowed & $33(2.6 \%)$ & $14(1.3 \%)$ & $47(2 \%)$ \\
\hline Work & Yes & $556(43.7 \%)$ & $660(61.6 \%)$ & $1,216(51.9 \%)$ \\
Situation & No & $652(51.2 \%)$ & $373(34.8 \%)$ & $1,025(43.7 \%)$ \\
& Never & $65(5.1 \%)$ & $38(3.6 \%)$ & $102(4.4 \%)$ \\
\hline
\end{tabular}

Trained psychologists interviewed participants individually using laptops containing an electronic version of each instrument used to collect data. The data set was encoded and then transferred to different statistical packages for data analysis. In the current investigation we used the AFECTS and the demographic questionnaire data.

The demographic questionnaire aimed to evaluate personal and social characteristics related to the sex, age, education and marital status, occupation, and other relevant information.

The AFECTS emotional section contains 48 items organized in five scales composed of 8 items (volition, anger, sensitivity, coping, and control) and two scales with 4 items each (fear and caution). The items are scored from 1 to 7 and the total score of each dimension is the sum of the scores of their respective items. ${ }^{1}$ In the current manuscript, we did not include the analysis of the AFFECTS affective section.

\section{Statistical analysis}

All analysis were performed using Mplus version 8.3 computer package ${ }^{14}$. Descriptive statistics related to demographic and temperament variables are presented using frequencies for categorical data and means and standard deviations (SDs) for continuous variables. Table 2 shows descriptive statistics regarding emotional traits. 
Table 2. Means and standard deviations for emotional trait scores

\begin{tabular}{|l|c|c|c|c|c|c|}
\hline Emotional Traits & \multicolumn{2}{|c|}{ Women } & \multicolumn{2}{c|}{ Men } & \multicolumn{2}{c|}{ Total } \\
\hline & Mean & SD & Mean & SD & Mean & SD \\
\hline Volition & 41.98 & 10.1 & 44.40 & 9.38 & 43.07 & 9.87 \\
\hline Anger & 32.51 & 11.47 & 28.41 & 10.71 & 30.63 & 11.32 \\
\hline Fear & 15.41 & 5.06 & 13.98 & 4.76 & 14.74 & 4.99 \\
\hline Caution & 19.13 & 5.91 & 19.76 & 5.77 & 19.42 & 5.86 \\
\hline Control & 43.11 & 9.72 & 43.46 & 9.77 & 43.26 & 9.77 \\
\hline Sensitivity & 32.89 & 10.45 & 27.63 & 9.61 & 30.50 & 10.42 \\
\hline Coping & 44.33 & 9.95 & 45.13 & 9.6 & 44.69 & 9.82 \\
\hline
\end{tabular}

Confirmatory factor analysis was used to test the a priori conceptual correlated 7 -factor model underlying the 48 categorical in-Likert format items that conformed AFECTS item set (fear and caution as independent factors). The weighted least square using a diagonal weight matrix with standard errors and mean- and variance-adjusted (WLSMV) estimator was used ${ }^{15}$, because the observed indicators (i.e., AFECTS items) have an ordinal-categorical format. Parameterization theta and probit link function were used. Moreover, due to the demographic sectors from which participants were retrieved (i.e., multilevel structure), the standard errors and chisquare test of the model fit took into account such non-independence following the procedures described by Asparouhov'16,17.

To evaluate the goodness of fit of the proposed 7-factor model, the following indices were used: Confirmatory Fit Indices (CFI), the Tucker-Lewis index (TLI), and root mean square error approximation (RMSEA). The cutoff criteria used to determine the goodness of fit are described as following: RMSEA estimate values near or less than 0.06 and RMSEA's close fit (Cfit) higher than 0.05 are indicator of appropriate model fit, while CFI and TLI values near or greater than 0.95 are considered indicate good model fit ${ }^{18}$. It is important to point out that CFI and TLI are penalized under complex models (i.e., multidimensional models with many items per factor and various factors), and such models, as proposed here, tend to worsen as the number of variables in the model increases ${ }^{19}$. Then, CFI and TLI's values near to 0.9 were considered to be indicative of good fit. Important to notice that Sivo et al. ${ }^{20}$, in a partial replication of $\mathrm{Hu}$ and Bentler's investigation ${ }^{18}$, showed that the cut-off values for goodness of fit coefficients must be decided considering different conditions such as model structure and sample size.

In terms of factor loading's magnitude, Nunnally ${ }^{21}$ asserts that it "is easy to overinterpret the meaning of small factor loadings, e.g., those below .40." Hence, we point estimate values for factor loadings values below 0.4 small magnitude effects.

Information curves were estimated for each factor. Trait level distribution is located at the $\mathrm{X}$-axis ( $\mathrm{z}$-scores) and the measurement of information is at the Y-axis. Values for information are not standardized: the higher the information scores in a given part of the trait spectrum, the higher the precision/reliability of the measure and, consequently, the test ability to capture reliably individual differences in a particular spectrum.

\section{Results}

The 7-correlated factor solution generated suitable model fit coefficient values for all observed indicators. The RMSEA estimate value was of 0.04 and its Cfit was equal to 1.0. The CFI and the TLI values were of 0.933 and 0.928 , respectively. Figure 1 portrays the correlated model depicting the standardized factor loading and the correlation among factors. Only one factor loading (FE3) showed a factor loading below of $0.4\left(\lambda_{\mathrm{FE} 3}=0.289\right.$, p-value $\left.<0.001\right)$ which correspond a reliability $\left(\mathrm{R}^{2}=8.35 \%\right)$.

Information curves showed that trait scores had particularities. Volition, caution, control and coping displayed the highest level of information at the trait spectrum around and below mean. Fear information curve was more distributed along trait spectrum, displaying the highest information parameter ranging from the $1^{\text {st }} \mathrm{SD}$ below and the $2^{\text {nd }} \mathrm{SD}$ above the mean score, with a low decrease after the $2^{\text {nd }} \mathrm{SD}$ above mean. Anger and sensitivity highest information level were situated in between the $1^{\text {st }} \mathrm{SD}$ below and $2^{\text {nd }} \mathrm{SD}$ above the mean scores. Figure 2 depicts information curves for each factor.

\section{Discussion}

The results showed herein attest to the robustness of the AFECTS emotional section as a reliable and valid tool for assessing temperament traits. The seven-factor latent structure presumed to underline the AFECTS item set displayed satisfactory goodness of fit index values and factor loadings were moderate to high, which indicated that theoretical traits accounted for a substantial portion of its items covariance. Information curve estimations showed that AFECTS trait scores measure reliably a wide range of its theoretical constructs. Taken together, results are coherentr with the AFECT conceptual framework ${ }^{1}$ and previous psychometric investigations of the AFECTS 1,8 , with one exception: in this study fear and caution were successfully designed as first-order factors.

The division of the Inhibition into two factors of fear and caution produced a valid general solution (a 7-factor solution for AFECTS emotional scale). This division is also supported by to previus empirical data that show that fear and caution stablish opposite relatioships with criteria variables such as substance misuse ${ }^{4}$ or traumatic experiences 6 . Moreover, fear and caution under our 7-correlated factor solution exhibited a very small standardized correlation $(r=0.285)$, which indicates a divergent validity between both domains.

Correlations among latent traits were also conceptually meaningful and similar in magnitude and direction to the ones reported in previous research ${ }^{1,8}$. Traits presumed to tap frontal functions and with desirable psychosocial adjustment content (i.e.,

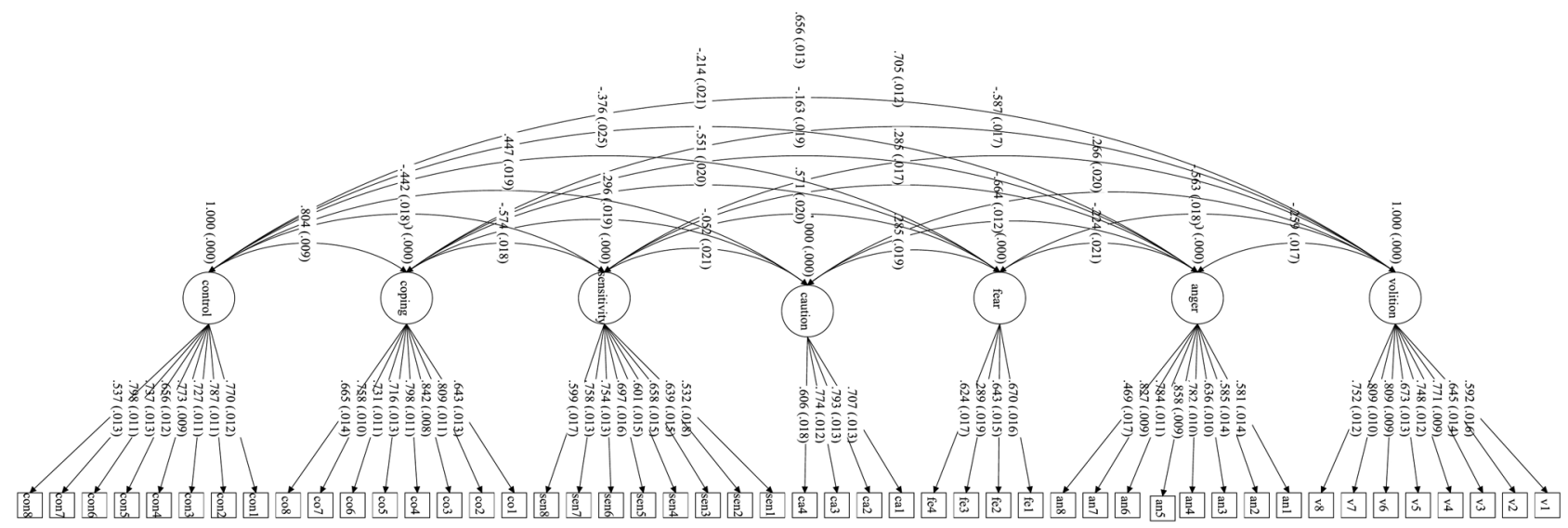

Figure 1. Seven-factor correlated model for AFECTS item set. 

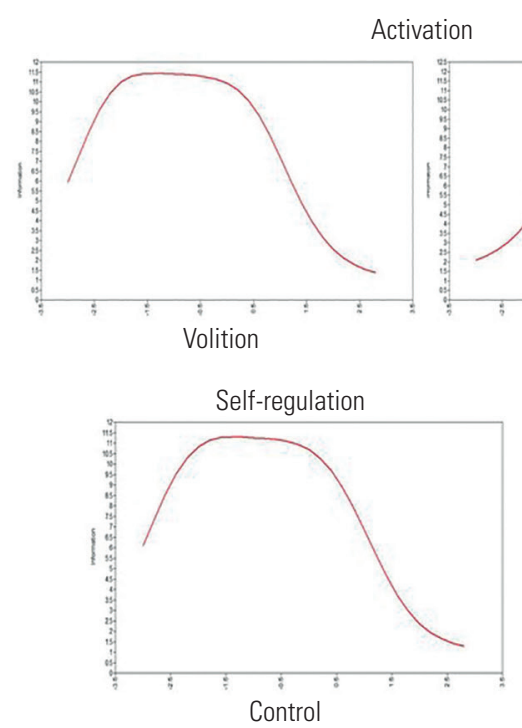

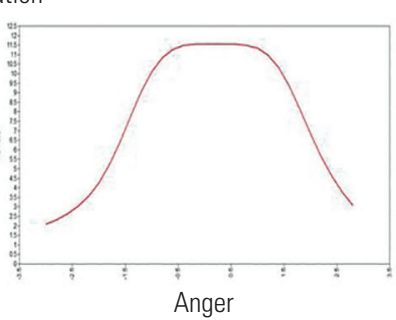

Anger

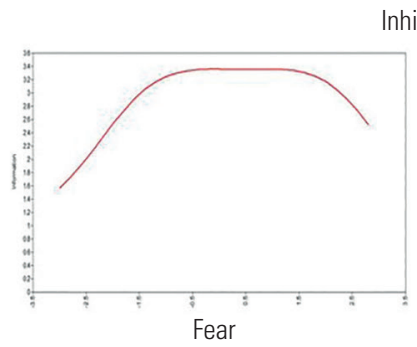

Inhibition

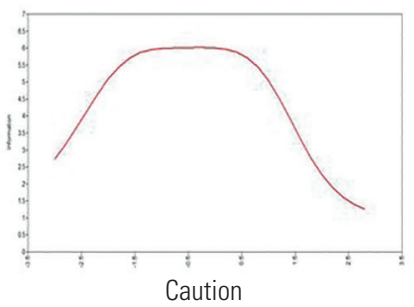

Caution

Figure 2. Information curves for AFECTS emotional traits.

volition, caution, control, and coping) displayed positive correlations with each other. Similarly, traits related to negative psychosocial adjustment content (such as anger, fear, and sensitivity) displayed positive correlations with each other. The traits fear and caution showed a small positive correlation $(r=0.28)$ as both may be conceived as inhibition processes: the first is associated with more innate and spontaneous reactions towards hazardous stimulations (such as freezing) and the second is related to more sophisticated processes of inhibition, based on the perception of environment cues that predict harmful events ${ }^{22}$. This poor empirical association and differences in function favor the understanding of fear an caution as independent factors.

Information curve analyses showed that AFECTS emotional section scores were more reliable to measure its underlying traits in individuals that are located between one to two standard deviations above and below mean. Volition, caution, control and coping are more reliable to assess individuals with average and low scores. Fear is more reliable to assess individuals located around the mean and both below and above mean scores, while sensitivity and anger are more reliable to evaluated mean and above mean scores. In general, AFECTS trait scores are less reliable to assess extreme trait manifestations: three standard deviations below and above mean score. These patterns have one particular implication: the AFECTS seem to be a reliable instrument to assess trait levels that tap the majority of the population (between 2 SDs below and above mean); which proves its reliability to evaluated normal-range temperament manifestations and individuals with subclinical or mild manifestations of various mood psychopathologies. Therefore, it is plausible to state that AFECT model offer relevant transdiagnostic variables ${ }^{23}$.

The current research has virtues and limitations worth of mention. The main virtues are related to the adopted sampling and analytical procedures: first, a randomized population-based sample maximizes the generalization power of our findings to the strata of individual with age ranging from 14 to 35 years. Second, this is the first study that evaluated the psychometrical properties of the AFECTS emotional section in a sample of adolescents, showing that the temperament constructs purported by the AFECT model are also present at this age spectrum. Third, the use of a modern psychometric approach to test model structural hypothesis and reliability of trait scores indicate the robustness of both: the theoretical model and its measurement tool. Nevertheless, face-to-face interviews and selfreport instruments also display its well-documented shortcomings ${ }^{24}$. Also, in this article we limited the analysis to the emotional section of AFECTS, evaluating the psychometric properties of AFECTs temperament trait assessment.

\section{Conclusion}

The current study shows the robustness of the AFECTS emotional sertion to assess temperament traits among adolecents and adults alike. The division of inhibition into two correlated factor of fear and caution yelded a stable factor solution.

\section{Individual contributions}

HWC and HC-M undertook the statistical analysis. HWC wrote the manuscript. DRL, KJ, RS, LS and JB designed the study and worked on the implementation of data collection procedures. All authors revised the manuscript.

\section{Disclosure}

The authors declare to have no conflict of interests.

\section{Ethics}

This study was approved by the committee of ethics in research from the Catholic University of Pelotas (UCPEL), under Protocol number $15 / 2010$.

\section{References}

1. Lara DR, Bisol LW, Brunstein MG, Reppold CT, de Carvalho HW, Ottoni GL. The affective and emotional composite temperament (AFECT) model and scale: a system-based integrative approach. J Affect Disord. 2012;140(1):14-37.

2. Lara DR, Akiskal HS. Toward an integrative model of the spectrum of mood, behavioral and personality disorders based on fear and anger traits: II. Implications for neurobiology, genetics and psychopharmacological treatment. J Affect Disord. 2006;94(1-3):89-103.

3. Lara DR, Pinto O, Akiskal K, Akiskal HS. Toward an integrative model of the spectrum of mood, behavioral and personality disorders based on fear and anger traits: I. Clinical implications. J Affect Disord. 2006;94(1-3):67-87.

4. Romo-Nava F, Fresán-Orellana A, Barragán V, Saracco-Álvarez R, Becerra-Palars C, Osorio Y, et al. The Affective and Emotional Composite Temperament Scale (AFECTS): Psychometric properties of the Spanish version in a community sample from Mexico City and comparison between remitted psychiatric patients. J Affect Disord. 2015;172:251-8.

5. Sudbrack R, Manfro PH, Kuhn IM, de Carvalho HW, Lara DR. What doesn't kill you makes you stronger and weaker: how childhood trauma relates to temperament traits. J Psychiatr Res. 2015;62:123-9. 
6. Guerrin LD, de Carvalho HW, Lara DR. The relationship between temperament and sexual orientation. J Affect Disord. 2015;175:379-84.

7. Fuscaldo LV, Bisol LW, Lara DR. How emotional traits and affective temperaments relate to cocaine experimentation, abuse and dependence in a large sample. Addict Behav. 2013;38(3):1859-64.

8. Leite L, Machado LN, Lara DR. Emotional traits and affective temperaments in alcohol users, abusers and dependents in a national sample. J Affect Disord. 2014;163:65-9.

9. Mombach KD, de Souza Brito CL, Padoin AV, Casagrande DS, Mottin CC. Emotional and Affective Temperaments in Smoking Candidates for Bariatric Surgery. 2016. PLoS One. 2016;11(3):e0150722.

10. Lara RD, Ottoni GL, Bisol LW, Carvalho HW. The integration of mood, behavior, and temperament in mood spectrum disorders. In: Chi-Kain LW, Gunderson JG, Orgs. Borderline Personality and Mood Disorders. 1st ed. New York: Springer; 2015; p. 133-54.

11. Ottoni GL, Antoniolli E, Lara DR. Circadian Preference Is Associated With Emotional and Affective Temperaments. Chronobiol Int. 2012;29(6):786-93.

12. Lara DR, Ottoni GL, Brunstein MG, Frozi J, de Carvalho HW, Bisol LW. Development and validity data of the Brazilian Internet Study on Temperament and Psychopathology (BRAINSTEP). J Affect Disord. 2012;141(2-3):390-8.

13. Birnbaum MH. Human research and data collection via the internet. Ann Rev Psychol. 2004;55:803-32.

14. Muthén LK, Muthén BO. Mplus User's Guide. 8th ed. Los Angeles, CA: Muthén \& Muthén; 1998-2018.
15. Muthén B, du Toit SHC, Spisic D. Robust inference using weighted least squares and quadratic estimating equations in latent variable modeling with categorical and continuous outcomes [Unpublished manuscript]. Los Angeles, CA: College of Education, UCLA; 1997.

16. Asparouhov T. Sampling Weights in Latent Variable Modeling. Struct Equ Modeling. 2005;12(3):411-34

17. Asparouhov T. General multi-level modeling with sampling weights. Communications in Statistics - Theory and Methods. 2006;35(3):439-60.

18. Hu L, Bentler PM. Cutoff criteria for fit indexes in covariance structure analysis: Conventional criteria versus new alternatives Struct Equ Modeling. 1999;6:1-55.

19. Kenny DA, McCouch DB. Effect of the Number of Variables on Measures of Fit in Structural Equation Modeling. Struct Equ Modeling. 2003;10:333-51.

20. Sivo SA, Xitao Fan E, Witta L, Willse JT. The Search for "Optimal" Cutoff Properties: Fit Index Criteria in Structural Equation Modeling, J Exp Educ. 2006;74: 267-88.

21. Nunnally J. Psychometric Theory. New York, NY: McGraw-Hill; 1967.

22. Sylvers P, Lilienfeld SO, LaPraire L. Differences between trait fear and trait anxiety: Implications for psychopathology. Clin Psychol Rev. 2011;31(1):122-37.

23. Patrick CJ, Rajcak G. RDoC: Translating promise into progress. Psychophysiology. 2016;53(3):415-24.

24. Conrad GF, Schober MF. New forntiers in standardized survey interviewing. In: Hesse-Biber SN, Leavy P. Handbook of Emergent Methods. 1st ed. New York: The Guilford Press; 2008. 